\title{
Editorial
}

\section{BNP: soon to become a routine measure in the care of patients with heart failure?}

Clinicians are increasingly using biochemical markers of disease to help diagnose and monitor disease states. Might B-type natriuretic peptide (BNP) become the same to heart failure as thyroid function tests are to hypothyroidism?

BNP is a 32 amino acid peptide secreted chiefly from the cardiac ventricles in response to ventricular strain. On secretion, proBNP, the storage form of BNP, is cleaved into the inactive $\mathrm{N}$-terminal-proBNP and the endocrinologically active BNP. The active moiety promotes natriuresis, diuresis, and vasodilatation. The blood concentration of BNP is raised in patients with cardiac disease, particularly those with heart failure.

Both plasma BNP and NT-proBNP can be rapidly measured by radioimmunoassay or immunoradiometric assay from an EDTA blood sample. Reliable near patient testing is also just around the corner. These technical advances have made the use of BNP in routine practice a much more practical proposal.

\section{BNP as an aid to the diagnosis of heart failure}

The reliability of a clinical diagnosis of heart failure is poor, especially in primary care. Recent guidelines have recommended that the clinical diagnosis of heart failure should be backed up by objective confirmation of underlying cardiac dysfunction. Echocardiography is the safest and most practical method of confirming dysfunction. However, there are at least 120000 suspected new cases of heart failure each year in the UK. If we are to advocate echocardiography for all such patients we must consider the resource requirements. In the UK most echocardiographic services are already working to capacity. Might the measurement of BNP help us to target imaging to those most likely to benefit from it?

In the Hillingdon heart failure study in west London, only 35 out of 122 referrals to a rapid access heart failure clinic, with a new diagnosis of heart failure from their primary care physician, had the diagnosis confirmed on further assessment. Limiting referral for assessment of possible heart failure to those patients with a plasma BNP concentration $>22 \mathrm{pmol} / \mathrm{l}$ would have reduced the number of patients assessed by more than a half, with $70 \%$ of those being assessed having the diagnosis confirmed and only one patient with heart failure being "missed". Confirmation of these data in larger studies in routine care should be available within the year.

Several groups have shown the value of measuring BNP concentration in patients admitted to hospital with acute breathlessness. Although the diagnosis of heart failure in such circumstances is not as difficult as when patients have milder symptoms, BNP can help discriminate between those with breathlessness caused by heart failure from those with breathlessness from other causes. ${ }^{2}$ BNP will never become a replacement for echocardiography. A normal BNP concentration virtually excludes left ventricular systolic dysfunction, but a high concentration merely indi- cates the presence of some cardiac problem which requires further investigation. ${ }^{3}$

\section{BNP as a prognostic marker for patients with heart failure}

Several reports suggest that the natriuretic peptides are important markers of the prognosis of patients with heart failure. Gottlieb and colleagues reported that the plasma concentration of atrial natriuretic peptide (ANP) was strongly and independently associated with survival in a series of 102 patients with chronic heart failure. ${ }^{4}$ Similar findings were reported from the neurohormonal substudy of the SAVE (survival and ventricular enlargement) study. ${ }^{5}$ In the general elderly population the plasma concentration of ANP also predicts cardiovascular mortality and the risk of heart failure. ${ }^{6}$ For theoretical reasons the plasma concentration of BNP is likely to be even more strongly associated with survival. Preliminary work from a large population based study of incident cases of heart failure in Bromley, south London, suggest that plasma BNP and proNT-BNP concentrations are both highly associated with survival and the risk of rehospitalisation. If these data are confirmed it is likely that clinicians will be requesting BNP measurement to help target more intensive treatment and follow up to patients most at risk of death or hospitalisation.

\section{A better way to assess control of the syndrome?}

Traditionally, the chronic monitoring of patients with heart failure has been rather crude. Increasing fluid retention is treated by adjustment in the dose of diuretic and other medication, provided hypotension does not supervene. Hospitalisation is often necessary to regain control of fluid retention. It would be useful to have a cheap biochemical marker of the control of the syndrome. Is BNP likely to be this marker? With the advent of near patient testing for BNP, it might be possible for a patient with heart failure to monitor their disease just as patients with diabetes check their blood sugar. After appropriate education many patients should be able to cope with adjusting their medication on the basis of such serial measurements. BNP, however, has a yet greater potential role. A small study from Glasgow has suggested that titration of angiotensin converting enzyme (ACE) inhibitor treatment with the aid of serial BNP measurements leads to a greater inhibition of the renin-angiotensin-aldosterone system and a significant fall in heart rate compared with empiric treatment. ${ }^{7}$ The benefits of this hormone guided approach over the conventional strategy in terms of reducing hospitalisation and death await the results of ongoing randomised controlled trials.

Interesting preliminary data from the use of carvedilol in patients with ischaemic left ventricular systolic dysfunction suggest that the benefits of $\beta$ blockade may be greatest in those with a high BNP concentration but no pronounced elevation of noradrenaline (norepinephrine) 
concentrations: neurohormonal profiling of patients may help us determine which of an increasingly long list of drugs may be of benefit to our patients. ${ }^{8}$

\section{Other possible uses}

There is an increasing literature supporting the use of BNP measurement after myocardial infarction. Not only does it identify those patients likely to have significant left ventricular systolic dysfunction (making it very useful in centres that cannot provide echocardiography for all their postinfarct patients), ${ }^{9}$ but it may be better than echocardiography at identifying those patients who are at high risk of progressive ventricular dilatation and remodelling, ${ }^{10}$ heart failure or death. ${ }^{11}$ Work from the Glasgow MONICA (monitoring trends and determinants in cardiovascular disease) study also suggests that BNP measurement might be a useful method of identifying individuals with significant but asymptomatic left ventricular systolic dysfunction in the general population. ${ }^{12}$

\section{Brave new world?}

Although more work is necessary, it is clear that BNP is rapidly moving from being a research tool to being a clinically useful test. In the near future, primary care physicians will be using BNP to help confirm a clinical diagnosis of heart failure and the need for further cardiac assessment. Cardiologists will use it to help assess prognosis and the need for more intensive treatment. Perhaps most excitingly we may start finding it of benefit in monitoring our patients with heart failure, using serial measurements to fine tune treatment in a more scientific way than we are able to do at present.

Cardiology Department,

Aberdeen Royal Infirmary

Aberdeen AB25 2ZD, UK

email:m.cowie@abdn.ac.uk

1 Cowie MR, Struthers AD, Wood DA, et al. Value of natriuretic peptides in assessment of patients with possible new heart failure in primary care. Lancet 1997;350:1349-51

2 Flesicher D, Espiner EA, Yandle TG, et al. Rapid assay of plasma brain natriuretic peptide in the assessment of acute dyspnoea. NZ Med $f$ 1997;110:71-4.

3 Struthers AD. How to use natriuretic peptide levels for diagnosis and prognosis. Eur Heart 7 1999;20:1374-5.

4 Gottlieb SS, Kukin ML, Ahern D, et al. Prognostic importance of atrial natriuretic peptide in patients with chronic heart failure. $\mathcal{f} \mathrm{Am}$ Coll Cardiol 1989:13:1534-9.

5 Vantrimpont P, Rouleau JL, Ciampi A, et al. Two-year time course and significance of neurohumoral activation in the survival and ventricular enlargement (SAVE) study. Eur Heart f 1998;19:1552-63.

6 Davis KM, Fish LC, Elahi D, et al. Atrial natriuretic peptide levels in the prediction of congestive heart failure in frail elderly. $7 A M A$ 1992;267: 2625-9.

7 Murdoch DR, McDonagh T, Byrne J, et al. Titration of vasodilator therapy in chronic heart failure according to plasma brain natriuretic peptide concentration: randomized comparison of hemodynamic and neuroendocrine effects of tailored versus empirical therapy. Am Heart f 1999;138: 1126-32.

8 Richards AM, Doughty R, Nicholls MG, et al. Neurohormonal prediction of benefit from carvedilol in ischemic left ventricular dysfunction. AustraliaNew Zealand heart failure group. Circulation 1999;99:786-92.

9 Cho Zuar tion A-MJ, Darbar D, Lang CC, et al. Detection of tion after acute myocardial infarctir

10 Arakawa N, Nakawura M, Aoki $\mathrm{H}$, et al. Relationships between plasma level of brain natriuretic peptide and myocardial infarct size. Cardiology 1994;85:334-40.

11 Richards AM, Nicholls MG, Yandle TG, et al. Neuroendocrine prediction of left ventricular function and heart failure after acute myocardial infarction. The Christchurch cardioendocrine research group. Heart 1999;81:114-20. 12 McDonagh T, Robb SD, Murdoch DR, et al. Biochemical detection of leftventricular systolic dysfunction. Lancet 1998;351:9-13.

\section{STAMPS IN CARDIOLOGY}

\section{Transplantation}
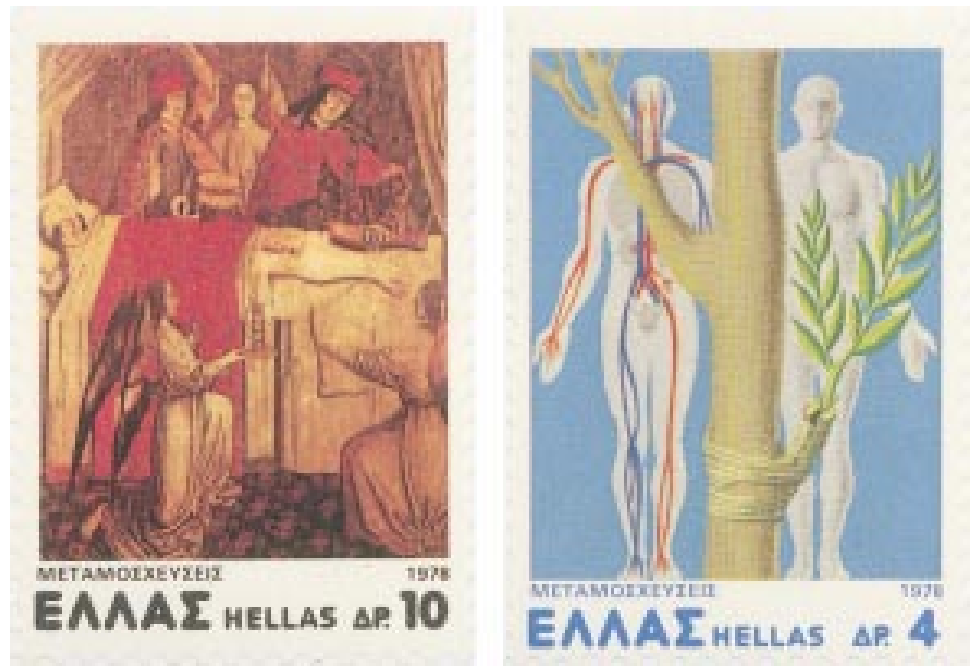

The 4 drachma 1978 stamp from Greece depicts a grafted plant and the diagram of the circulation. The other stamp in the set commemorating transplants is the famous painting by Alonso de Sedano "The Miracle of Sts Cosmas and Damian" where the leg of a white man has been amputated and transplanted with one from a black man.

M K DAVIES A HOLLMAN 